\title{
Incidence of Mucoid Degeneration of Cruciate Ligaments in Patients Undergoing Primary Total Knee Replacements - A Study of 42 Knees
}

\author{
Rajanish R R $^{\mathrm{a}}$, Harisankar Ma, Jim Thomas Malayila, Muhammad P K ${ }^{\mathrm{a}}$, Ani Praveen \\ a. Department of Orthopaedics, Baby Memorial Hospital, Calicut, Kerala, India; \\ b. Department of Pathology, Baby Memorial Hospital, Calicut, Kerala, India
}

Published on $1^{\text {st }}$ June 2020

\begin{abstract}
Mucoid degeneration of the ACL is largely an underdiagnosed condition. These are often misjudged as partial tears of ACL on routine MRI evaluation. Here we histologically analyse the incidence of mucoid degeneration of the cruciate ligaments in 32 patients who underwent primary total knee arthroplasty in our institute. 10 among them had bilateral total knee replacements making the total sample size 42 knees.

ACL samples were sent for histopathological evaluation from 41 knees (one knee was an ACL deficient knee), and PCL samples were sent from patients who underwent total knee replacements with posterior stabilising implants. out of these 41 samples of ACL, $60.9 \%$ turned out to be positive for mucoid degeneration. 9 out of 13 PCL samples $(69.5 \%)$ turned out to be having mucoid degeneration.

This study shows that the real incidence of mucoid degeneration of cruciate ligaments is much more than what is reported in literature. This emphasises the role of preoperative MRI to look for coexisting painful mucoid degeneration even in elderly patients with early osteoarthritis, which is an arthroscopically treatable condition thus delaying the total knee replacements in these patients. The higher incidence of mucoid degeneration in cruciate ligaments associated with advanced arthritis raises the question of functional integrity of PCL in these patients, making the use of posterior stabilising implants more advisable in patients with advanced stages of osteoarthritis.
\end{abstract}

Key Words: Mucoid Degeneration, ACL, PCL, Total Knee Replacement, Cruciate Retaining, Posterior Stabilising, MRI

\section{INTRODUCTION}

$\mathrm{M}$ ucoid degeneration of the anterior cruciate ligament (ACL) was first reported by Kumar et al in $1999 .{ }^{1}$ Even though it is a rarely reported condition, recent studies show that the incidence of mucoid degeneration is higher than that was initially thought. ${ }^{2}$ These degenerations are often misjudged as partial ligament tears. The etiology of the mucoid degeneration remains unclear, but the studies have proved an invariable association to old age. ${ }^{3}$ And even in situations where the ACL is intact on macroscopic evaluation, there may be histological changes that question the integrity of the ligament. ${ }^{4}$
Here we put forward a study of histological examination of cruciate ligaments from 42 knees after total knee arthroplasty in our hospital, either with cruciate retaining (CR) or posterior stabilising (PS) implants.

\section{LITERATURE REVIEW}

The first case of mucoid degeneration of ACL was reported by Kumar et al in 1999. ${ }^{1}$ Since then, many case reports have been published regarding the etiology, histology and biomechanical implications of the mucoid degeneration of the cruciate ligaments. In a study of 4221 knees by Bergin et $\mathrm{al},{ }^{5}$ they found that the condition was far more frequent than was initially thought.

Cite this article as: R R, M H, Malayil JT, K MP, Praveen A. Incidence of Mucoid Degeneration of Cruciate Ligaments in Patients Undergoing Primary Total Knee Replacements - A Study of 42 Knees. KJOrth. 2020 Jun 1;33(01):2-7.

Corresponding Author:

Dr M Harisankar, Department of Orthopaedics Baby Memorial Hospital, Calicut, Kerala, India. Mobile: 09037081225 E-mail: drharisankarm@gmail.com
Access this Article Online

$$
\text { Scan this QR Code }
$$

https:/t/doi.org/10.52314/kjo.2020.v33i01.4

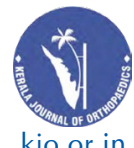

kjo.or.in 


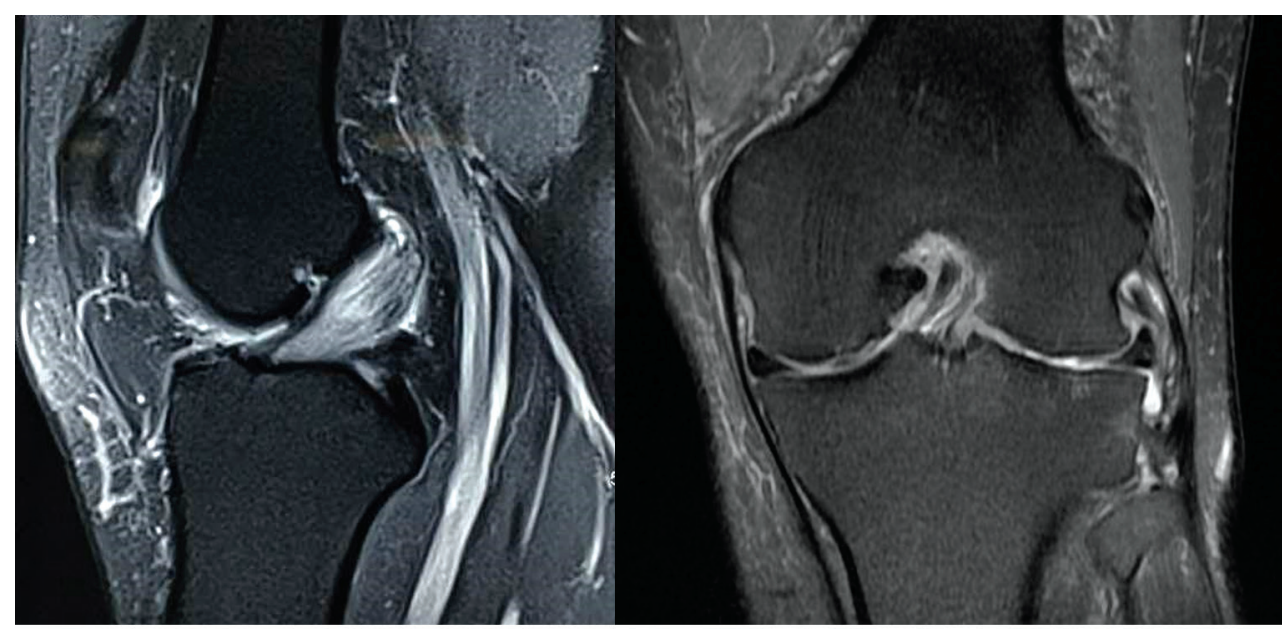

Figure 1. T2 weighted MRI images (sagittal and coronal cuts) showing celery stalk appearance of ACL

\section{Imaging}

Gold standard for diagnosis of mucoid degeneration of ACL is Magnetic Resonance Imaging (MRI). Classical MRI findings include intermediate signal intensity in $\mathrm{T} 1$ $(2,7-9)$ weighted images and an increased signal intensity in T2 weighted images. The ligament will appear thick, wavy and hypertrophied. The mucoid substance is seen intermingled with the collagen fibres dissecting between them, but not interrupting them. The typical appearance of ACL in mucoid degeneration was described as "Celery stalk" appearance (Figure 1,2) by many authors. ${ }^{2,9-11}$

Radiological differential diagnosis include a partial tear of the ACL, ganglion cyst or even a malignant process. ${ }^{2}$ Shelly et al has reported case of metastatic adenocarcinoma of the lung into the knee mimicking mucoid degeneration of the ACL. ${ }^{12}$

\section{Arthroscopic findings}

It is characteristically described as bulky ACL with decreased vascularity filling the intercondylar notch. The yellowish tinge of mucoid degeneration can be seen on macroscopic examination with the naked eye. Most of them are nonfunctional.

Another common finding is the absence of synovial lining of the ACL. McIntyre et al in their study proposed an arthroscopic diagnostic criteria for ACL mucoid degeneration. $^{13}$

\section{ACL fibres intact}

2. Increased ACL diameters

3. Yellow substance found by probing

4. Loss of synovial lining

\section{Pathophysiology}

One popular theory explaining the pathogenesis of mucoid degeneration is its temporal association with trivial trauma. Glycosaminoglycan, that contributes to the mucoid substance is secreted by the ACL fibroblast in response to trauma. $^{2}$

Another theory puts forward the existence of ectopic synovial tissue within the ACL giving rise to mucoid degen- 
eration. ${ }^{10}$ Kim et al in their study proposes that mucoid degeneration of ACL and mucoid cystic degeneration of the ACL presents a pathogenetic continuum. This study also shows association of ACL mucoid degeneration to osteoarthritis of the knee. ${ }^{14}$

\section{Histology}

The lesions contain yellow or brown thick or dense basophilic substances containing high density of glycosaminoglycans (hyaluronic acid). Hyaluronic acid is detected by staining with Alcian Blue stain. ${ }^{15}$

\section{Mucoid degeneration of PCL}

Mucoid degeneration of the PCL is even less frequently reported than mucoid degeneration of ACL. Kleinbert et $\mathrm{al}^{15}$ in their study, stated that in severe knee arthritis, PCL displays disorganisation of the parallel collagen fiber bundles. This collagen disorganisation and loss of integrity of the ligaments have been found to alter the functionality of the ligaments in significant animal studies. ${ }^{16,17}$ A study of histopathological analysis of the PCL by Martins et al ${ }^{18}$ in 2018 showed only a small percentage of patients with advanced arthritis retained PCL with a functional neurovascular bundle.

\section{MATERIALS AND METHODS}

\section{Estimation of sample size}

Sample size estimation considering $\mathrm{p}<0.05$ as acceptable significance and study with $80 \%$ power

$$
\mathrm{n}=\left\{(1.96)^{2} \times \mathrm{p} \times \mathrm{q}\right\} / \mathrm{d}^{2}
$$

As per the reference study, ${ }^{19}$ mucoid degeneration was observed in $53.6 \%$ of the study population.

Hence,

$$
\begin{aligned}
& \mathrm{p}=53.6 \% \\
& \mathrm{q}=100-\mathrm{p}=46.4 \% \\
& \mathrm{~d}=\text { precision }=15 \%
\end{aligned}
$$

Thus, $\mathrm{n}=42.4$, rounded off as 42 patients.

Patients who underwent primary total knee arthroplasty in our institute was included in the study. 10 of them had bilateral total knee replacements, making the sample size a total of 42 knees. Out of these, 34 knees had Kellgren and Lawrence ${ }^{20}$ stage 4 osteoarthritic changes and 8 knees had stage 3 osteoarthritis on preoperative radiographic evaluation. They all underwent Total knee replacement with either CR or PS implants. The decision to retain or to sacrifice the PCL was made based on preoperative clinical examination and the intra-operative macroscopic examination of the integrity of PCL.

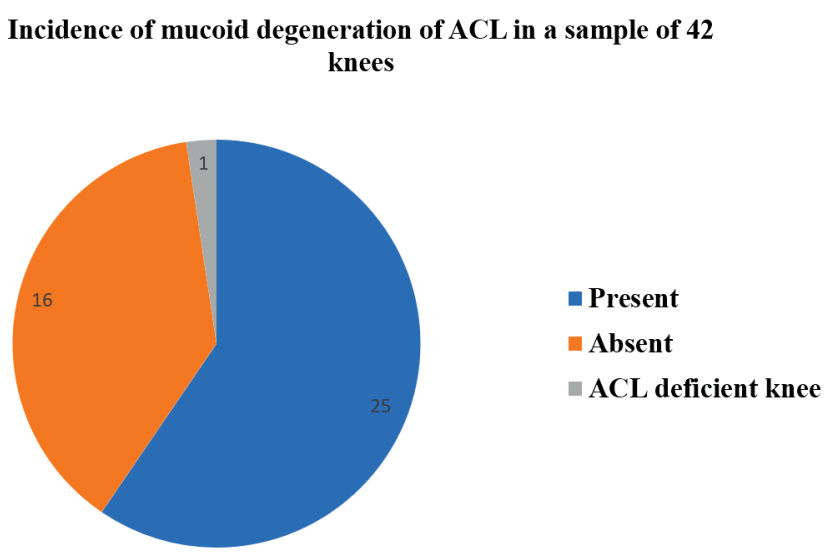

Figure 3. Chart showing incidence of mucoid degeneration of ACL in a sample of 42

ACL from every case and PCL from patients who underwent Total knee replacement with PS prosthesis was sent for histopathological evaluation. All specimens were immediately preserved in formalin solution and was sent for histopathological examination as per routine laboratory practice.

\section{RESULTS}

- The sample consisted of 32 patients with a mean age of 63.5 years. 23 patients $(71 \%)$ were females. 27 patients suffered from Osteoarthritis and 5 patients had Rheumatoid arthritis.

- 34 knees had Kellgren and Lawrence ${ }^{20}$ stage 4 osteoarthritic changes and 8 knees had stage 3 osteoarthritis changes

- 10 patients underwent bilateral TKR, making the total sample size 42 knees.

- 29 (69\%) underwent TKR using cruciate retaining implants and the rest $13(31 \%)$ underwent posterior stabilising TKR.

- ACL from all the knees were sent for histopathological examination, except for 1 case in which no ACL was seen intraoperatively (ACL deficient knee). PCL was sent for histopathological examination from 13 patients who underwent TKR with posterior stabilising implants.

- Out of the 41 samples of ACL that was sent for histopathological examination, $25(60.9 \%)$ turned out to be positive for mucoid degeneration (Figure 3). Histological picture was characteristic with fibrocollagenous tissue with myxoid degeneration (Figures 4 A,B).

- Out of 13 PCL samples sent for histopathological evaluation, $9(69.2 \%)$ had changes suggestive of mucoid degeneration (Figure 5)

- All the 9 knees which had mucoid degeneration of PCL showed mucoid degeneration in their ACL samples too. 


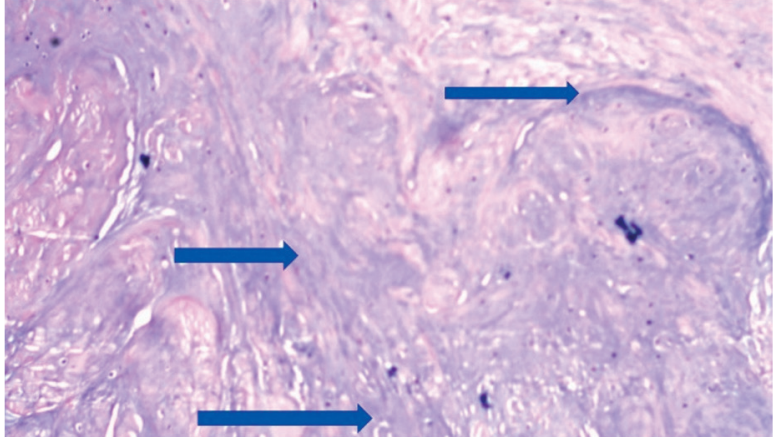

Figure 4A.

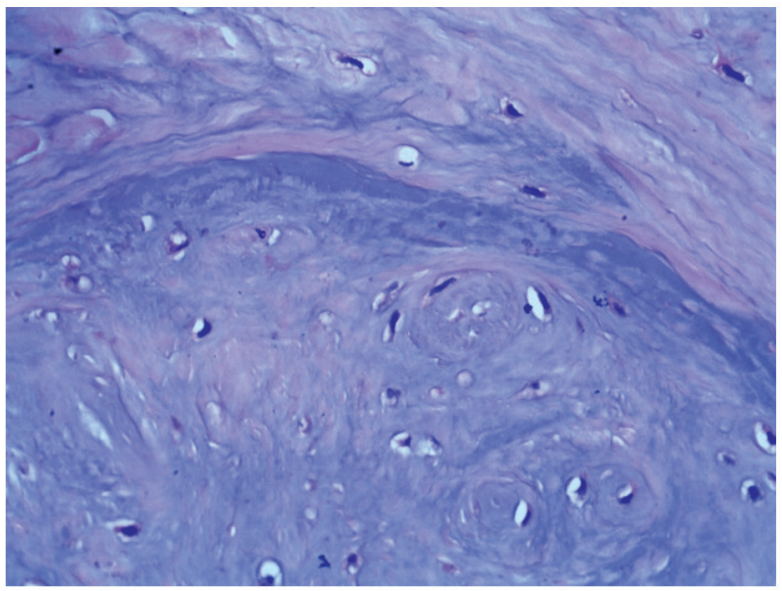

Figure 4B.

Figure 4. Histopathology section shows fibrocollagenous tissue with myxoid degeneration (A) H\&E X100, and (B) H\&E X400

This is in accordance with studies by Allain et $\mathrm{al}^{21}$ and Albert et $\mathrm{al}^{22}$ who proposed mucoid degeneration in the ACL gives way to mucoid degeneration in the PCL.

- Out of the remaining 4 knees, 2 had isolated mucoid degeneration of ACL alone.

- No cases of isolated mucoid degeneration of PCL was seen in the present study.

- Out of 10 patients who underwent bilateral TKR, 6 had CR implants in both knees, 3 had PS implants in both knees and 1 had CR implant in one knee and PS implant in the other. Among the histopathological results of these 10 patients, 2 patients had unilateral mucoid degeneration in ACL.

\section{DISCUSSION}

It has been proposed that the suboptimal results in some patients after total knee replacement (TKR) is due to the failure to obtain normal knee biomechanics after the surgery. The drive these days has been to preserve as much of the native knee as possible. With advent of unicondylar knee and ACL sparing total knee prosthesis, the functional status of the cruciate ligaments come into question.

This study has highlighted the fact that mucoid degenera- tion of the ACL is either an underdiagnosed or misdiagnosed condition. Many ACL specimens which appeared normal in macroscopic evaluation turned out to be having mucoid degeneration on microscopic evaluation. Allain and Gouttalier et $\mathrm{al}^{21}$ in their study reported a correlation between ACL and PCL degeneration. Several studies have been published showing clear association of degenerative changes in the PCL with advanced osteoarthritis. As per literature, $85 \%$ of knees with severe osteoarthritis show degeneration of the PCL. ${ }^{21,23}$

The purpose of preferring the CR prosthesis over PS prosthesis is to ensure proper femoral roll back during flexion of the knee and to enhance proprioception through retaining the PCL. Arthroplasty with PCL preservation demands not only a structural preservation of the ligament, but also functional preservation of its proprioceptive capacity. ${ }^{18,21}$ Nelisen et $\mathrm{al}^{23}$ in their study proposes that the mucoid degeneration of the PCL mirrors the radiological arthritic status of the knee and advocates the use of PS implant in knees with advanced stages of arthritis even when the PCL is clinically intact. Allain et $\mathrm{al}^{21}$ and Albert et $\mathrm{al}^{22}$ in their studies reached the same conclusion that there was a higher incidence of degeneration in PCL in patients who had ACL degeneration. There is no evidence in the literature as to whether the mucoid degeneration of the PCL will further progress after cruciate retaining TKR restoring the normal biomechanics of the knee. In this study group, out of 13 samples, 9 turned out to be histologically positive for mucoid degeneration of PCL. This is a significant number, thus warranting further evaluation in this direction and a bigger study with a higher sample size.

With this observation of higher numbers of histological degeneration of PCL than that was previously thought, the logical conclusion would be to choose PS over CR implants in patients with advanced osteoarthritis. But data shows high patient satisfaction even with CR implants. These satisfactory results despite an insufficient PCL could be explained by the design and conformity of the implants and by the fact that the ligaments take on only a secondary role in the containment when the implant is submitted to axial loading. ${ }^{24}$

\section{Incidence of mucoid degeneration of PCL from 13 samples}

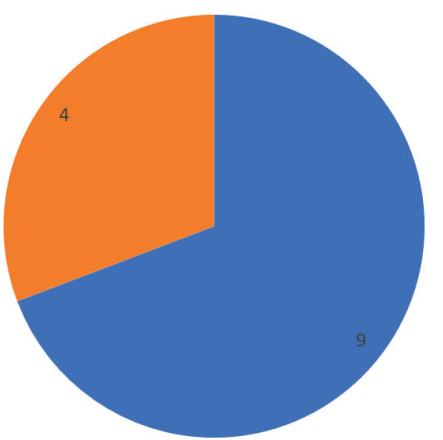

- Present Absent

Figure 5. Chart showing incidence of mucoid degeneration of PCL from 13 samples 
MRI with cartilage study remains the gold standard in preoperatively diagnosing the mucoid degeneration of the cruciate ligaments and assessing the concurrent stage of osteoarthritis. The characteristic features in MRI is termed "celery stalk" appearance. The other features include an intermediate intensity signal in T1 weighted images and a high intensity signal in T2 weighted images. Preoperative diagnosis of mucoid degeneration of the cruciate ligaments help in planning the procedure better. Not to ignore the fact that mucoid degeneration in itself is a neurogenic pain generating condition associated with progressively decreased range of movement of knee mimicking the symptoms of severe osteoarthritis of the knee. ${ }^{25}$ In patients with early osteoarthritis of knee, intractable pain and restriction of range of movements, the diagnosis of coexisting mucoid degeneration of the cruciate ligaments should be entertained. Mucoid degeneration, once proven with MRI, can be treated with minimally invasive arthroscopic procedures like ACL debridement and notchplasty or total ACL reconstruction, thus delaying TKR in young patients and even in old patients with early stage Osteoarthritis. MRI of the knee with cartilage study can also correctly grade the tricompartmental osteoarthritis status.

\section{CONCLUSION}

Mucoid degeneration of the cruciate ligaments is an underdiagnosed condition. The real incidence of mucoid degeneration is much higher than what has been already published in the literature.

There is a clear role for preoperative MRI of the knee with cartilage study in patients undergoing total knee replacements to look for mucoid degeneration in the cruciate ligaments and to assess the cartilage status of the articular surfaces. This will be of help especially when clinical findings and Xray findings do not match. This might allow for arthroscopic treatment of the condition thus delaying the TKR in patients of all age groups, and also help in avoiding postoperative patient dissatisfaction after total knee replacement. Preoperative MRI evaluation can give surgeon an insight to decide if the PCL should be retained or sacrificed during total knee replacement.

The decision to retain or sacrifice the posterior cruciate ligament during TKR in patients without preop MRI should not be based on clinical and intra-operative macroscopic examination of the integrity of the ligament alone, but also on the stage of arthritis and the degree of radiographic destruction of knee which parallels the histological degeneration of the cruciate ligaments.

If intraoperatively there are macroscopic features suggestive of mucoid degeneration of the ACL in patients with advanced osteoarthritis, it is better to select posterior stabilising implants than to retain the PCL, as in these patients, the chances of degeneration of PCL is very high.
This might lead to postoperative reduction of knee range of movements and can also cause postoperative posterior knee pain.

\section{END NOTE}

\section{Author Information}

1. Rajanish R, Department of Orthopaedics, Baby Memorial Hospital, Calicut, Kerala, India.

2. Harisankar M, Department of Orthopaedics, Baby Memorial Hospital, Calicut, Kerala, India.

3. Jim Thomas Malayil, Department of Orthopaedics, Baby Memorial Hospital, Calicut, Kerala, India.

4. Muhammad P K, Department of Orthopaedics, Baby Memorial Hospital, Calicut, Kerala, India.

5. Ani Praveen, Department of Pathology, Baby Memorial Hospital, Calicut, Kerala, India.

Conflict of Interest: None declared

\section{REFERENCES}

1. Kumar A, Bickerstaff DR, Grimwood JS, Suvarna SK. Mucoid cystic degeneration of the cruciate ligament. J Bone Joint Surg Br. 1999 Mar;81(2):304-5.

2. Lintz F, Pujol N, Boisrenoult P, Bargoin K, Beaufils P, Dejour D. Anterior cruciate ligament mucoid degeneration: a review of the literature and management guidelines. Knee Surg Sports Traumatol Arthrosc. 2011 Aug; 19(8):1326-33.

3. Kawaguchi K, Michishita K, Manabe T, Akasaka Y, Arakawa T, Higuchi J. Mucoid degeneration of the cruciate ligaments in osteoarthritis under primary total knee arthroplasty. Asia Pac J Sports Med Arthrosc Rehabil Technol. 2018 Apr;12:1-4.

4. Mont MA, Elmallah RK, Cherian JJ, Banerjee S, Kapadia BH. Histopathological Evaluation of the Anterior Cruciate Ligament in Patients Undergoing Primary Total Knee Arthroplasty. J Arthroplasty. 2016 Jan;31(1):284-9.

5. Bergin D, Morrison WB, Carrino JA, Nallamshetty SN, Bartolozzi AR. Anterior cruciate ligament ganglia and mucoid degeneration: coexistence and clinical correlation. AJR Am J Roentgenol. 2004 May;182(5):1283-7.

6. Salvati F, Rossi F, Limbucci N, Pistoia ML, Barile A, Masciocchi C. Mucoid metaplastic-degeneration of anterior cruciate ligament. J Sports Med Phys Fitness. 2008 Dec;48(4):483-7.

7. Choubey R, Jain A. Mucoid Degeneration of the Anterior Cruciate Ligament: A Case Report and Review of Literature. J Orthop Case Rep. 2015 Sep;5(3):87-9.

8. Matrawy KA, El-Nekeidy AEAM, Al-Dawody A. Mucoid degeneration of the anterior cruciate ligament: Frequently under-diagnosed entity in MRI. Egyptian Journal of Radiology and Nuclear Medicine. 2012;43(2):227-33.

9. Fernandes JL, Viana SL, Mendonça JLF, Freitas FMO, Bezerra ASA, Lima G -a. S, et al. Mucoid degeneration of the anterior cruciate ligament: magnetic resonance imaging findings of an underdiagnosed entity. Acta Radiol. 2008 Feb;49(1):75-9.

10. Hensen JJ, Coerkamp EG, Bloem JL, De Schepper AM. Mucoid degeneration of the anterior cruciate ligament. JBR-BTR. 2007 Jun;90(3):192-3.

11. Hsu C-J, Wang S-C, Fong Y-C, Huang C-Y, Chiang I-P, Hsu H-C. Mucoid degeneration of the anterior cruciate ligament. J Chin Med Assoc. 2006 Sep;69(9):449-52. 
12. Shelly MJ, Dheer S, Kavanagh EC. Metastatic adenocarcinoma of the lung mimicking mucoid degeneration of the anterior cruciate ligament. Irish journal of medical science. 2010;179(2):309-311.

13. McIntyre J, Moelleken S, Tirman P. Mucoid degeneration of the anterior cruciate ligament mistaken for ligamentous tears. Skeletal Radiol. 2001 Jun;30(6):312-5.

14. Kim T-H, Lee D-H, Lee S-H, Kim J-M, Kim C-W, Bin S-I. Arthroscopic treatment of mucoid hypertrophy of the anterior cruciate ligament. Arthroscopy. 2008 Jun;24(6):642-9.

15. Kleinbart FA, Bryk E, Evangelista J, Scott WN, Vigorita VJ. Histologic comparison of posterior cruciate ligaments from arthritic and age-matched knee specimens. J Arthroplasty. 1996 Sep;11(6):726-31.

16. Bosch U, Decker B, Möller HD, Kasperczyk WJ, Oestern HJ. Collagen fibril organization in the patellar tendon autograft after posterior cruciate ligament reconstruction. A quantitative evaluation in a sheep model. Am J Sports Med. 1995 Apr;23(2):196-202.

17. Oxlund H, Andreassen TT. The roles of hyaluronic acid, collagen and elastin in the mechanical properties of connective tissues. J Anat. 1980 Dec;131(Pt 4):611-20.

18. Martins GC, Camanho G, Rodrigues MI, Filho LFM, Demange MK. Histopathological analysis of the posterior cruciate ligament in primary osteoarthritis. Eur J Orthop Surg Traumatol. 2018 May;28(4):691-9.
19. Youm Y-S, Cho S-D, Cho H-Y, Jung S-H. Relationship between Mucoid Degeneration of the Anterior Cruciate Ligament and Posterior Tibial Slope in Patients with Total Knee Arthroplasty. Knee Surg Relat Res. 2016 Mar;28(1):34-8.

20. Kohn MD, Sassoon AA, Fernando ND. Classifications in Brief: Kellgren-Lawrence Classification of Osteoarthritis. Clin Orthop Relat Res. 2016 Aug;474(8):1886-93.

21. Allain J, Goutallier D, Voisin MC. Macroscopic and histological assessments of the cruciate ligaments in arthrosis of the knee. Acta Orthop Scand. 2001 Jun;72(3):266-9.

22. Albert A, Forthomme J-P, Vandenhooft A, Van Eeckhout P, Feoli F. Are lesions of the posterior cruciate ligament predictable before knee arthroplasty? A histological study of 434 ligaments in osteoarthritic knees. Acta Orthop Belg. 2008 Oct;74(5):652-8.

23. Nelissen R, Hogendoorn P. Retain or sacrifice the posterior cruciate ligament in total knee arthroplasty? A histopathological study of the cruciate ligament in osteoarthritic and rheumatoid disease. J Clin Pathol. 2001 May;54(5):381-4.

24. Keyes GW, Carr AJ, Miller RK, Goodfellow JW. The radiographic classification of medial gonarthrosis. Correlation with operation methods in 200 knees. Acta Orthop Scand. 1992 Oct;63(5):497-501.

25. Pandey V, Suman C, Sharma S, Rao SP, Kiran Acharya K, Sambaji C. Mucoid degeneration of the anterior cruciate ligament: Management and outcome. Indian J Orthop. 2014;48(2):197-202. 